\title{
Cosmological constraints from a 2D SZ catalog
}

\author{
S. Mei ${ }^{1,2}$ and J. G. Bartlett ${ }^{3}$ \\ 1 Institut d'Astrophysique Spatiale, UMR-8617, Université Paris-Sud, Bâtiment 121, 91405 Orsay Cedex, France \\ 2 Johns Hopkins University, 3400 N. Charles Street, 21218 Baltimore, MD, USA \\ e-mail: smei@pha.jhu.edu \\ 3 APC - Université Paris 7, 11 place Marcelin Berthelot, 75005 Paris, France \\ e-mail: bartlett@cdf.in2p3.fr
}

Received 9 October 2003 / Accepted 7 May 2004

\begin{abstract}
We perform a Fisher matrix analysis to quantify cosmological constraints obtainable from a 2-dimensional Sunyaev-Zel'dovich (SZ) cluster catalog using the counts and the angular correlation function. Three kinds of SZ survey are considered: the almost all-sky Planck survey and two deeper ground-based surveys, one with $10 \%$ sky coverage, the other one with a coverage of 250 square degrees. With the counts and angular function, and adding the constraint from the local X-ray cluster temperature function, joint $10 \%$ to $30 \%$ errors $(1 \sigma)$ are achievable on the cosmological parameter pair $\left(\sigma_{8}, \Omega_{\mathrm{M}}\right)$ in the flat concordance model. Constraints from a 2D distribution remain relatively robust to uncertainties in possible cluster gas evolution for the case of Planck. Alternatively, we examine constraints on cluster gas physics when assuming priors on the cosmological parameters (e.g., from cosmic microwave background anisotropies and SNIa data), finding a poor ability to constrain gas evolution with the 2-dimensional catalog. From just the SZ counts and angular correlation function we obtain, however, a constraint on the product between the present-day cluster gas mass fraction and the normalization of the mass-temperature relation, $T_{*}$, with a precision of $15 \%$. This is particularly interesting because it would be based on a very large catalog and is independent of any X-ray data.
\end{abstract}

Key words. cosmology: cosmic microwave background - cosmology: cosmological parameters - galaxies: clusters: general

\section{Introduction}

Over the coming few years, surveys of galaxy clusters observed with the Sunyaev-Zel'dovich (SZ) effect (Sunyaev \& Zel'dovich 1970, 1972; Birkinshaw 1999; Carlstrom et al. 2002) will open a new observational window onto large-scale structure formation and evolution (Barbosa et al. 1996; Eke et al. 1996; Colafrancesco et al. 1997; Diego et al. 2002; Haiman et al. 2001; Holder et al. 2001; Kneissl et al. 2001; Weller et al. 2002; Benson et al. 2002). The advantages offered by this window, as compared to either the X-ray or the optical, are intrinsic to the SZ effect (Bartlett 2000). They include the ability to detect clusters at high redshift, due to the lack of surface brightness dimming in the SZ, and a "clean" selection on cluster gas thermal energy, a robust quantity expected to have a tight relationship to cluster mass. These properties are particularly advantageous for evolutionary studies because they permit the selection of similar mass clusters over a large range of redshifts. The distribution of cluster abundance with redshift, for example, is sensitive to the cosmological parameters $\sigma_{8}$ and $\Omega_{\mathrm{M}}$, and also, although less so, to $\Omega_{\Lambda}$ and the dark energy equation-of-state (Oukbir \& Blanchard 1997; Barbosa et al. 1996; Haiman et al. 2001). This potential is currently motivating a number of observational efforts aimed at realizing
SZ surveys with dedicated, optimized interferometers (AMI ${ }^{1}$, $\mathrm{AMiBA}^{2}, \mathrm{SZA}^{3}$ ), and large-format bolometer arrays $\left(\mathrm{APEX}^{4}\right.$, $\mathrm{ACT}^{5}, \mathrm{BOLOCAM}^{6}, \mathrm{ACBAR}^{7}, \mathrm{SPT}^{8}$ ). The Planck ${ }^{9}$ satellite, to be launched in 2007, will provide a full-sky catalog of galaxy clusters detected by their SZ signal, one of the largest galaxy cluster catalogs ever constructed, and in the more distant future one may look forward to an even larger catalog from a fourth generation CMB mission, such as the Inflation Probe proposed by NASA in the context of the Beyond Einstein Program ${ }^{10}$.

Follow-up in other wavebands of a SZ catalog is obviously essential for many scientific goals, for instance to constrain cosmology and cluster evolution with the redshift distribution and X-ray properties (e.g., Holder et al. 2001;

\footnotetext{
${ }^{1}$ http://www.mrao.cam.ac.uk/telescopes/ ami/index.html

${ }^{2}$ http://www.asiaa.sinica.edu.tw/amiba

${ }^{3}$ http://astro.uchicago.edu/sze

${ }^{4}$ http://bolo.berkeley.edu/apexsz

5 http://www.hep. upenn. edu/ angelica/act/act.html

${ }^{6}$ http://astro.caltech.edu/ lgg/bolocam_front.htm

${ }^{7}$ http://cosmology. berkeley.edu/group/swlh/acbar/

${ }^{8}$ http://astro.uchicago.edu/spt/

9 http://astro.estec.esa.nl/Planck/

${ }^{10}$ http://universe.nasa.gov/program/probes.html
} 
Table 1. The surveys that have been considered in this analysis.

\begin{tabular}{ccccc}
\hline \hline Survey & $Y$ limit $\left(\right.$ arcmin $\left.^{2}\right)$ & Coverage (sq. deg.) & Average redshift & $\begin{array}{c}\text { Expected number of clusters } \\
\left(\text { for } \Omega_{\mathrm{M}}=0.27, \Omega_{\Lambda}=0.73, h=0.72, \sigma_{8}=0.84\right)\end{array}$ \\
\hline Planck & $3 \times 10^{-4}$ & 40000 & 0.3 & 36000 \\
SPT & $5 \times 10^{-5}$ & 4000 & 0.6 & 33000 \\
APEX & $2.5 \times 10^{-5}$ & 250 & 0.7 & 5000 \\
\hline
\end{tabular}

Bartelmann \& White 2002; Diego et al. 2002; Weller et al. 2002; Hu 2003; Majumdar \& Mohr 2003a,b). Extensive follow-up will be limited to only small subsets of the larger SZ catalogs. In particular, the follow-up of the Planck all-sky catalog will represent a significant effort. It is therefore interesting to ask the question as to what science can be done with a two-dimensional SZ catalog, what we refer to as the SZ photometric catalog.

In a previous paper (Mei \& Bartlett 2003, MB03), we studied the counts and the angular correlation function of SZ clusters to see how these two statistics could be combined to extract cosmological information before any subsequent follow-up. The angular function has been extensively studied by Diaferio et al. (2003), while three dimensional clustering issues are elaborated by Moscardini et al. (2002). Specifically, we explored how joint measurements of the counts and angular function could be used to constrain the cosmological parameters $\sigma_{8}$ and $\Omega_{\mathrm{M}}$, when the normalization of the mass-temperature relation for clusters is known. This work focused on the influence of various cosmological parameters and cluster gas physics on both the counts and the angular function. In previous work, Fan $\&$ Chiueh (2001) examined constraints in the $\sigma_{8}-\Omega_{\mathrm{M}}$ plane obtained by combining a SZ catalog with limited redshift information (e.g., only two redshift bins) and the local abundance of $\mathrm{X}$-ray clusters.

We extend our study in this paper by quantifying the achievable constraints with a standard Fisher analysis, working in the context of the so-called concordance model $\left(\Omega_{\mathrm{M}}=0.27\right.$, $\Omega_{\Lambda}=0.73, h=0.72$; e.g. Spergel et al. 2003). Two kinds of constraints are used to illustrate the use of a SZ photometric catalog: constraints on the cosmological parameter pair $\left(\sigma_{8}, \Omega_{\mathrm{M}}\right)$ in the presence of possible cluster gas evolution, and constraints on cluster gas physics assuming strong cosmological priors (e.g., from cosmic microwave background anisotropies and SNIa distance measurements). We furthermore examine the gain obtained by incorporating the constraint from the local X-ray cluster temperature distribution function.

With just the photometric catalog, we find that determinations of $\sim 10 \%$ on $\sigma_{8}$ and $\sim 25 \%$ on $\Omega_{\mathrm{M}}$ are possible, assuming reasonable uncertainty on cluster gas physics. On the other hand, gas evolution is only poorly constrained even when adopting strong cosmological priors. Our present study takes as examples the almost full-sky SZ catalog expected from Planck, (Aghanim et al. 1997; Bartelmann 2001; Diego et al. 2002, and references therein), and two deeper ground-based experiments, one covering 4000 square degrees (e.g., Haiman et al. 2001; Holder et al. 2001; Majumdar \& Mohr 2003b), representative of the South Pole Telescope (SPT) survey, and one covering 250 square degrees, representative of the APEX survey. The characteristics of these surveys are summarized in Table 1 with flux limits quoted at a signal-to-noise of better than three. The expected number of clusters and mean redshift have been calculated for the case of our fiducial model (see Sect. 4).

In the next section we outline our cluster model, referring for details to MB03. The Fisher analysis is then presented in Sect. 3. Section 4 presents our main results in terms of achievable constraints on $\sigma_{8}$ and $\Omega_{\mathrm{M}}$ and on cluster gas physics. A final discussion follows.

\section{SZ cluster physics}

We give only a brief summary of our main modeling ingredients, leaving greater detail to MB03. Properties of the cluster population reflect the influence of halo evolution and cluster gas physics. For the halos, we adopt the mass function of Sheth $\&$ Tormen (1999) and use the expression for the linear growth factor $D\left(z, \Omega_{\mathrm{M}}, \Omega_{\Lambda}\right)$ given by Carroll et al. (1992). Halo clustering is modeled with a linear bias, $b(M, z)$, described by the analytic fit of Sheth et al. (2001). We use the BBKS transfer function with shape parameter fixed at $\Gamma=0.25$ for the matter density perturbation power spectrum. Although not critical for our final results, we include non-linear evolution of the density field according to the prescription developed by Peacock \& Dodds (1996).

The total SZ flux from a cluster (relative to the mean sky brightness, i.e., the unperturbed cosmic microwave background $(\mathrm{CMB})$ ) is measured by the integrated Compton $y$-parameter, which may be expressed in terms of cluster quantities as

$Y(M, z)=\frac{k \sigma_{T}}{m c^{2}} \frac{N_{\mathrm{e}} T}{D_{\mathrm{ang}}^{2}(z)} \propto \frac{f_{\mathrm{gas}}(M, z) T(M, z) M}{D_{\text {ang }}^{2}(z)}$

where $k$ is the Boltzmann constant, $m$ is the electron mass, and $N_{\mathrm{e}}$ is the total number of electrons in the cluster. In this expression, $f_{\text {gas }}(M, z)$ is the cluster gas mass fraction, $T(M, z)$ is the mean particle weighted gas temperature, $M$ is the total virial mass and $D_{\text {ang }}(z)$ is the angular diameter distance in a homogeneous background. Notice that $Y$ is directly proportional to the gas thermal energy, and we would expect this to have a tight relationship to cluster mass and redshift. This is indeed borne out by hydrodynamical simulations (da Silva et al. 2004), confirming the idea that SZ selection is "clean" and robust.

The gas mass fraction and temperature are in general functions of cluster mass and redshift, and we have taken care to write them as such. A number of simple scaling relations may be obtained by assuming that clusters form a self similar population, as would be expected if non-gravitational forces are 
sub-dominant (Kaiser 1986; Bryan \& Norman 1998). Such scaling arguments lead one to expect

$T(M, z)=T_{*}\left(M_{15} h\right)^{2 / 3}\left[\Delta(z) E(z)^{2}\right]^{1 / 3}\left[1-2 \frac{\Omega_{\Lambda}(z)}{\Delta(z)}\right]$

where $T_{*}$ is a normalization constant (expressed in $\mathrm{keV}$ ), $M_{15}$ is the cluster total mass in units of $10^{15} M_{\odot}, \Delta(z)$ is the non-linear density contrast on virialization $(\approx 178)$ and $h \equiv H_{\mathrm{o}} / 100 \mathrm{~km} \mathrm{~s}^{-1} \mathrm{Mpc}^{-1}$. The quantity $E^{2}(z)=\left[\Omega_{\Lambda}+\right.$ $\left.\left(1-\Omega_{\mathrm{M}}-\Omega_{\Lambda}\right)(1+z)^{2}+\Omega_{\mathrm{M}}(1+z)^{3}\right]$ (the dimensionless Hubble parameter) with the definitions $\Omega_{\mathrm{M}}(z) \equiv \Omega_{\mathrm{M}}(1+z)^{3} / E^{2}(z)$, $\Omega_{\Lambda}(z) \equiv \Omega_{\Lambda} / E^{2}(z)$; notice that $\Omega_{\mathrm{M}}$ and $\Omega_{\Lambda}$ written without an explicit redshift dependence will indicate present-day values $(z=0)$. The gas mass fraction $f_{\text {gas }}(M, z)$ is, on the other hand, constant in the simplest self-similar model, independent of cluster mass and redshift (e.g., Arnaud et al. 2002).

Putting all this together, we express the relation between cluster SZ flux and mass and redshift as

$Y(M, z)=Y_{15}(z) M_{15}^{5 / 3+\alpha}(1+z)^{\gamma}$

where $Y_{15}(z)$ incorporates the various constants and redshift dependence of the self-similar model. The exponents $\alpha$ and $\gamma$ describe any deviations from pure self-similarity, in other words gas evolution, such that the self-similar model is defined by $\alpha=\gamma=0$. In their cooling hydrodynamical simulations, da Silva et al. (2004) actually find very little deviation from selfsimilarity, even down to very low masses: $\alpha \approx 0.1$ and $\gamma \approx 0$. The explicit expression for $Y_{15}(z)$ is

$$
\begin{aligned}
Y_{15}(z)= & \left(7.4 \times 10^{-5} h^{7 / 6} \operatorname{arcmin}^{2}\right)\left(\frac{T_{*}}{\mathrm{keV}}\right)\left(\frac{f_{\mathrm{gas}}}{0.07 h^{-3 / 2}}\right) \\
& \times\left(\frac{\Delta(z) E(z)^{2}}{178}\right)^{1 / 3}\left[1-2 \frac{\Omega_{\Lambda}(z)}{\Delta(z)}\right] \frac{1}{d_{\mathrm{ang}}^{2}(z)} \\
\equiv & Y_{*}\left(\frac{\Delta(z) E(z)^{2}}{178}\right)^{1 / 3}\left[1-2 \frac{\Omega_{\Lambda}(z)}{\Delta(z)}\right] \frac{1}{d_{\mathrm{ang}}^{2}(z)} \\
\equiv & \left(1.06 \times 10^{-3} h^{8 / 3} \operatorname{arcmin}^{2}\right) Y_{*}^{\prime} \\
& \times\left(\frac{\Delta(z) E(z)^{2}}{178}\right)^{1 / 3}\left[1-2 \frac{\Omega_{\Lambda}(z)}{\Delta(z)}\right] \frac{1}{d_{\mathrm{ang}}^{2}(z)}
\end{aligned}
$$

where $D_{\text {ang }} \equiv H_{\mathrm{o}}^{-1} d_{\text {ang. }}$. In the following, we use $Y_{*}^{\prime} \equiv f_{\text {gas }} T_{*}$ to indicate our normalization of this relation. A certain amount of uncertainty remains in this constant due to uncertainties in both $T_{*}$ and $f_{\text {gas }}$. We will explore the effects of this uncertainty as well as of gas evolution by treating $\alpha, \gamma$ and $Y_{*}^{\prime}$ as free parameters constrained within observational limits.

\section{Fisher matrix method}

To calculate confidence regions we use the Fisher matrix method (e.g., Eisenstein et al. 1999; Holder et al. 2001), which is faster than Monte Carlo simulations and gives accurate enough results when the likelihood distribution is close to Gaussian. This is the case for the source counts that will number in the thousands (see Table 1). Since the angular correlations of SZ clusters are small on the scales of interest to us (e.g., tens of arcmins) and for the relevant survey depths (Diaferio et al. 2003, MB03), the statistical measurement error on the angular correlation function $w(\theta)$ can be modeled by the Poissonian variance in the number of random pairs $n_{\text {pair }}$ at separation $\theta$ in a ring $\Delta \theta$ (e.g., Peebles 1980; Landy \& Szalay 1993). As discussed in MB03, this quantity is determined by the cluster counts and equals $n_{\text {pair }} \approx(1 / 2) N \times\langle n\rangle=N \pi \theta \Delta \theta \Sigma$, $N$ being the total number of clusters in the catalog, and $\Sigma$ the surface density of clusters at the flux limit (cluster counts at the flux limit). In this case, and when the angular correlation function is measured by optimal estimators (Landy \& Szalay 1993), $w(\theta)$ can be treated as a Gaussian random variable with variance equal to its Poissonian value.

The Fisher matrix is defined as

$F_{i j} \equiv-\left\langle\frac{\partial^{2} \ln \mathcal{L}}{\partial p_{i} \partial p_{j}}\right\rangle=\left\langle\frac{\partial X}{\partial p_{i}} \frac{\partial X}{\partial p_{j}} \frac{1}{\sigma_{X}^{2}}\right\rangle$

where $\mathcal{L}$ is the likelihood for the physical variable $X$ and the $p_{i}$ are the parameters we wish to constrain; the angled brackets indicate an ensemble average over all possible data realizations. In our case $X$ will be respectively equal to the total number of clusters at the survey flux limit, $N$, and $w(\theta=30 \mathrm{arcmin})$ (see MB03 for this choice of separation); their respective errors are $\sigma_{N}^{2}=N$ and $\sigma_{w(\theta)}^{2}=\frac{1}{n_{\text {pair }}}$. We take the inverse of $F_{i j}$ to model the best covariance matrix $C_{i j}$ for the considered parameters, and we use Gaussian statistics (like $\chi^{2}$ ) to obtain one and two $\sigma$ confidence regions. We sum the two Fisher matrices to obtain the joint confidence limits from the combined measurement of both the counts and the angular correlation function. When incorporating the constraints from the X-ray temperature function, we add the three Fisher matrices. We have used singular value decomposition (Press et al. 1992) for every Fisher matrix to control singularities due to degeneracy among parameters.

\section{Results}

We consider two types of study to illustrate the uses of a photometric SZ catalog. In the first case, we are interested in constraining cosmological parameters, such as $\sigma_{8}$ and $\Omega_{\mathrm{M}}$ (for the flat concordance model with $h=0.72$ ), with just the two-dimensional catalog and in the presence of a certain amount of unknown cluster gas physics. As a second illustration, we assume the cosmological parameters are given and study the achievable constraints on the cluster gas physics. This might represent a study in which it is assumed that the cosmology is already fixed by other observations, such as measurements of CMB anisotropies and the SNIa Hubble diagram. We examine the three types of survey listed in Table 1.

\subsection{Constraints on $\sigma_{8}$ and $\Omega_{\mathrm{M}}$}

To get a feeling for the complementarity of the total counts and the angular function, we first examine a self-similar model with $\alpha=\gamma=0$, but with varying $Y_{*}^{\prime}$. The effects of gas evolution (i.e., deviations from self-similarity with $\alpha, \gamma \neq 0$ ) are studied in the second subsection. 

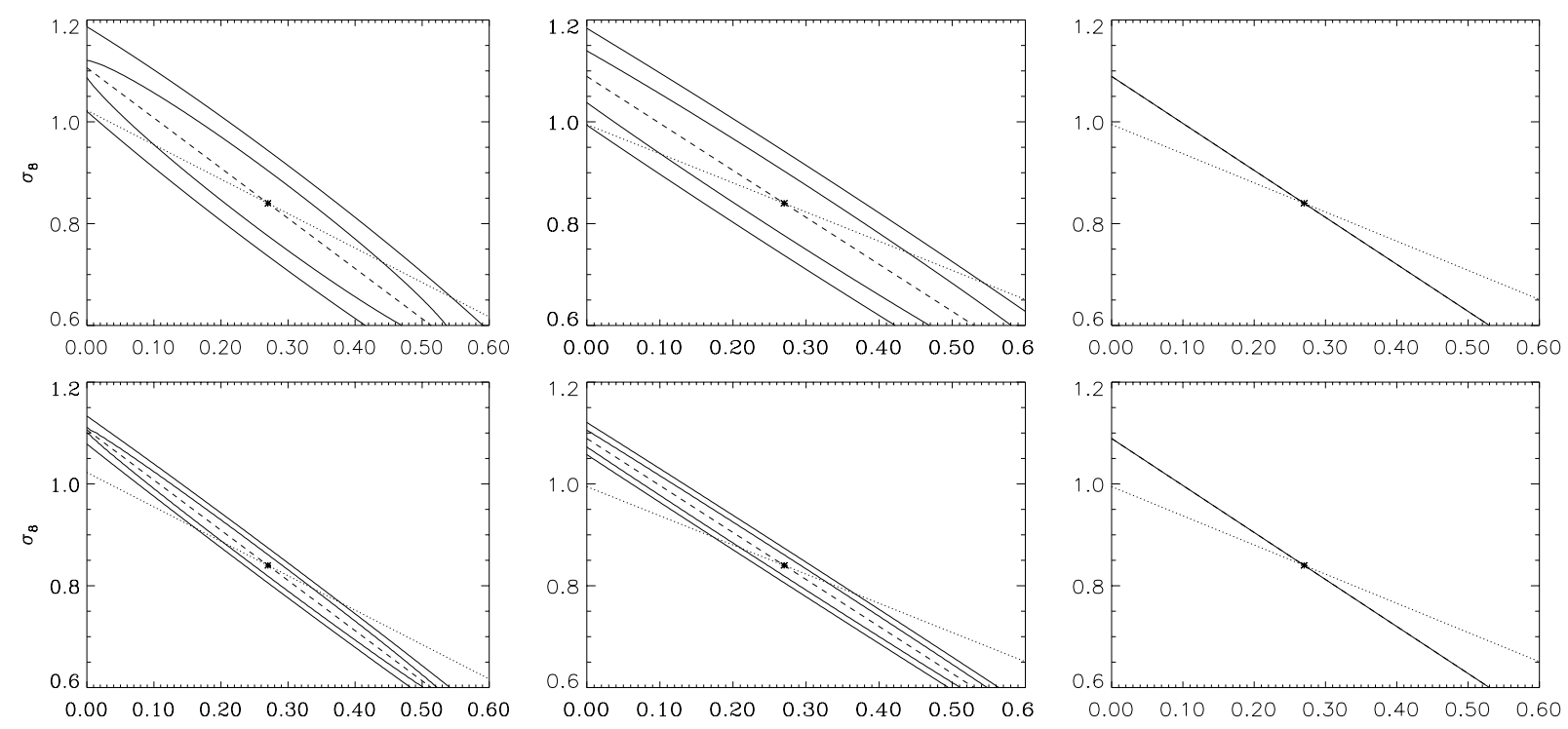

Fig. 1. Joint constraints from the counts and the angular correlation function on $\sigma_{8}$ and $\Omega_{\mathrm{M}}$ are shown (solid lines) at one and two $\sigma$ (2 independent parameters), with a prior of $30 \%$ (top) and $10 \%$ (bottom) on $Y_{*}^{\prime}$. Our fiducial model is shown as the asterisk. The dashed line represents the degeneracy line from the singular value decomposition of the count Fisher matrix. The dotted line represents the degeneracy line from the singular value decomposition of the angular correlation function Fisher matrix. From left to right we show the constraints for a Planck-like survey, a SPT-like survey and an APEX-like survey (see Table 1). In the case of the APEX-like survey, joint constraints are not shown, since the joint Fisher matrix is singular: in this case the solid line represents the degeneracy line from the singular value decomposition of the joint Fisher matrix; it falls directly on the degeneracy line from the the counts Fisher matrix.

\subsubsection{Constraints in the absence of gas evolution}

In Fig. 1 we show constraints in the $\sigma_{8}-\Omega_{\mathrm{M}}$ plane, marginalized over the free parameter $Y_{*}^{\prime}=f_{\text {gas }} T_{*}$. Our fiducial model adopts $Y_{*}^{\prime}=0.17 \mathrm{keV}$ - for example, $f_{\mathrm{gas}}=0.07 h^{-1.5}$ (Mohr et al. 1999; see also Grego et al. 2002) and $T_{*}=1.5 \mathrm{keV}$ (Pierpaoli et al. 2003) - and a flat cosmological model with $\sigma_{8}=0.84$ and $\Omega_{\mathrm{M}}=0.27$ (Spergel et al. 2003). Current values for $T_{*}$, whether from simulations or observational estimates, range from $1 \mathrm{keV}$ to about $2 \mathrm{keV}$ (Pierpaoli et al. 2003; Muanwong et al. 2002; Huterer \& White 2002; Finoguenov et al. 2001; Xu et al. 2001; Nevalainen et al. 2000; Horner et al. 1999), although we may hope that it will be much better known by the time large SZ catalogs become available, say with more thorough lensing studies. We therefore take as a representative possibility an uncertainty of $30 \%$ on $T_{*}$ and $10 \%$ on $f_{\text {gas }}$ (e.g., Mohr et al. 1999; Grego et al. 2002), which leads to an overall prior on $Y_{*}^{\prime}$ on the order of 30\% (top panel). A hopeful case would be to reach an overall uncertainty of $10 \%$ (bottom panel) on $Y_{*}^{\prime}$.

The dashed line represents the degeneracy line from the singular value decomposition of the total counts Fisher matrix. The dotted line represents the degeneracy line from the singular value decomposition of the angular correlation function Fisher matrix. The joint constraints from the total counts and angular correlation function are shown by the continuous contours at one and two sigma, and our fiducial model is indicated by the asterisk. Each catalog statistic is individually highly degenerate, but as discussed at length in $\mathrm{MB} 03$, the respective dependence of the two measures on $\sigma_{8}$ and $\Omega_{\mathrm{M}}$ differ, thereby lifting the degeneracies in the case of the Planck survey.

From left to right we show the constraints for a Plancklike, a SPT-like and an APEX-like survey. In the case of an
APEX-like survey, joint constraints are not shown because the joint Fisher matrix is singular: in this case the solid line represents the degeneracy line from the singular value decomposition of the joint Fisher matrix. It lies directly on the degeneracy line from the singular value decomposition of the counts Fisher matrix.

Since the counts and the angular correlation function depend on $\sigma_{8}, \Omega_{\mathrm{M}}$, and $Y_{*}^{\prime}$, it is not possible to constrain all three parameters without additional information. In Fig. 1 this information was taken as the prior on $Y_{*}^{\prime}$. As discussed in MB03, in fact the predicted curves for the counts and angular function shift around in the $\sigma_{8}-\Omega_{\mathrm{M}}$ plane with $Y_{*}^{\prime}$. Adding the local cluster abundance constraint pins down a unique point in this plane, thereby fixing both the cosmological parameters and the value of $Y_{*}^{\prime}$. The local abundance of clusters is quantified by the $\mathrm{X}$-ray temperature function and leads to a constraint on the parameter combination $\sigma_{8} \Omega_{\mathrm{M}}^{0.6} \approx 0.6 T_{*}^{-0.8}$ (Pierpaoli et al. 2003, and references therein). To apply this constraint, we need some prior information on $T_{*}$, for example from lensing or detailed X-ray studies. With such a prior, a joint analysis of the counts, angular function and local cluster abundance yields constraints on $\sigma_{8}$, $\Omega_{\mathrm{M}}$ and $Y_{*}^{\prime}$; the latter then also implies a constraint on $f_{\mathrm{gas}}$.

A joint analysis on $\sigma_{8}$ and $\Omega_{\mathrm{M}}$ from the counts, the angular function and the X-ray temperature function constraint, as taken from Pierpaoli et al. (2003), is shown Fig. 2. From left to right we show the constraints for Planck-like, SPT-like and APEX-like surveys. In the top panel, priors of $30 \%$ on $T_{\text {* }}$ and $50 \%$ on $f_{\text {gas }}$ are assumed. In the middle panel, priors of $10 \%$ on $T_{*}$ and $50 \%$ on $f_{\mathrm{gas}}$, and the bottom panel, priors of $10 \%$ on $T_{*}$ and $15 \%$ on $f_{\text {gas }}$ are assumed. Addition of the local abundance constraint tends to close the ends of the error ellipses. These results are summarized in Table 2 . We assume that 

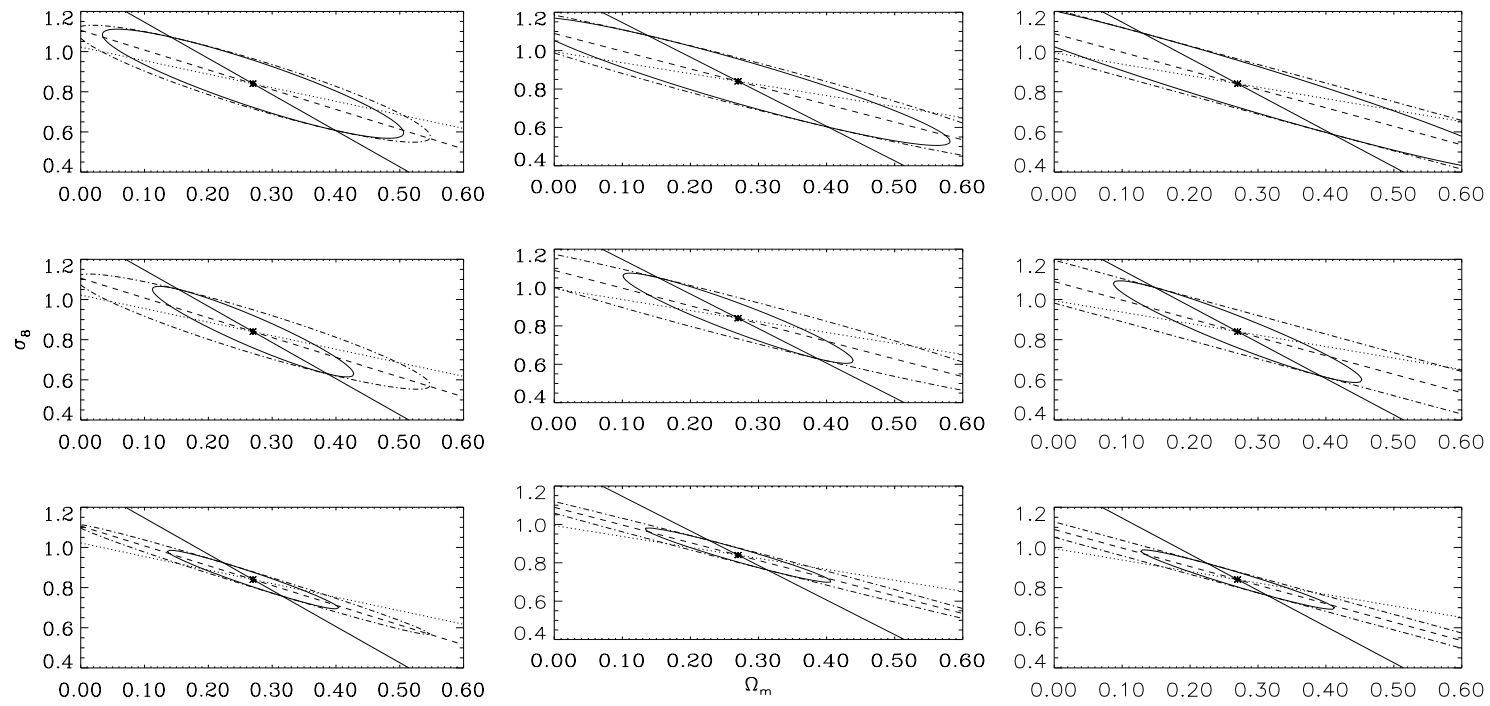

Fig. 2. Constraints on $\sigma_{8}$ and $\Omega_{\mathrm{M}}$ from a joint analysis of the counts, the angular function and the local X-ray temperature function (constraint from Pierpaoli et al. 2003) are shown at one $\sigma$ (continuous ellipse). The constraints from the joint counts and angular correlation function are shown by the dashed-dotted ellipse. The dashed line represents the degeneracy line from the singular value decomposition of the counts Fisher matrix. The dotted line represents the degeneracy line from the singular value decomposition of the angular correlation function Fisher matrix. The continuous line crossing the contours represents the degeneracy line from the singular value decomposition of the Fisher matrix for the constraints from the local X-ray temperature function. In the top panel, priors of $30 \%$ on $T_{*}$ and $50 \%$ on $f_{\text {gas }}$ are assumed; in the middle panel, priors of $10 \%$ on $T_{*}$ and $50 \%$ on $f_{\mathrm{gas}}$, and in the bottom panel, priors of $10 \%$ on $T_{*}$ and $15 \%$ on $f_{\mathrm{gas}}$ are assumed. From left to right, we show the constraints for Planck-like, SPT-like and APEX-like surveys. These constraints are summarized in Table 2.

Table 2. One sigma constraints on $\sigma_{8}$ and $\Omega_{\mathrm{M}}$ from a joint analysis of the counts, the angular function and the local X-ray temperature function. For each survey, the prior uncertainties on $T_{*}$ and $f_{\mathrm{gas}}$, and the expected final constraints on $\sigma_{8}, \Omega_{\mathrm{M}}$, and $f_{\mathrm{gas}}$ are given. From the joint analysis we derive constraints $\sigma_{8}, \Omega_{\mathrm{M}}$, but also gain precision on $f_{\text {gas }}$. This table summarizes the results of Fig. 2 .

\begin{tabular}{cccccc}
\hline \hline Survey & $T_{*}$ Prior unc. (\%) & $f_{\text {gas }}$ Prior unc. $(\%)$ & $\sigma_{\sigma_{8}}(\%)$ & $\sigma_{\Omega_{\mathrm{M}}}(\%)$ & $\sigma_{f_{\text {gas }}}(\%)$ \\
\hline Planck & 30 & 50 & 20 & 60 & 30 \\
& 10 & 50 & 20 & 40 & 30 \\
& 10 & 15 & 10 & 30 & 10 \\
\hline \multirow{2}{*}{ SPT } & 30 & 50 & 30 & 80 & 35 \\
& 10 & 50 & 20 & 40 & 30 \\
& 10 & 15 & 10 & 30 & 10 \\
\hline APEX & 30 & 50 & 35 & 100 & 35 \\
& 10 & 50 & 20 & 45 & 35 \\
& 10 & 15 & 10 & 35 & 10 \\
\hline
\end{tabular}

we already have some prior information on $f_{\text {gas }}$, corresponding to the actual observational situation. The fact that the joint analysis leads to an independent constraint on $f_{\text {gas }}$ is demonstrated by the gain in precision on this parameter shown in the last column of the Table. We conclude therefore that $10 \%-30 \%$ precision is possible on the cosmological parameters $\sigma_{8}$ and $\Omega_{\mathrm{M}}$ with a photometric SZ catalog and we emphasize that these constraints will be independent and complementary to those from Supernovae Ia and CMB measurements.

\subsubsection{Gas evolution}

We now examine the effects of deviations from gas selfsimilarity by varying $\alpha$ and $\gamma$ in addition to $Y_{*}^{\prime}$ (Eq. (3)). At present, very little can be said observationally concerning the exponents $\alpha$ and $\gamma$. For a discussion from an observational point of view see for example Ettori et al. (2003), and references therein. In their simulations, da Silva et al. (2004) find only small deviations from self-similarity, characterized by $\alpha \approx 0.1$ and $\gamma \approx 0$, down to very small cluster masses (well below $10^{14} M_{\odot}$ ) and in the presence of gas cooling. This illustrates the expected robustness of the SZ flux: as a measure of the total gas energy, determined largely by gravitational collapse, $Y$ remains rather insensitive to the details of the gas physics. The non-zero value of $\alpha$ reflects the change in gas thermal energy due to cooling, which will clearly have a greater effect on the lower mass systems. The majority of clusters in the Planck catalog will be rich systems (due to the high flux limit) that are relatively insensitive to heating/cooling mechanisms. Based on these considerations, we examine cosmological constraints with priors on $\alpha, \gamma$ of \pm 0.1 and \pm 1 , the latter most likely representing an extreme case.

Figure 3 shows the constraints on $\sigma_{8}$ and $\Omega_{\mathrm{M}}$ when including these new free parameters. Our fiducial model is once again 

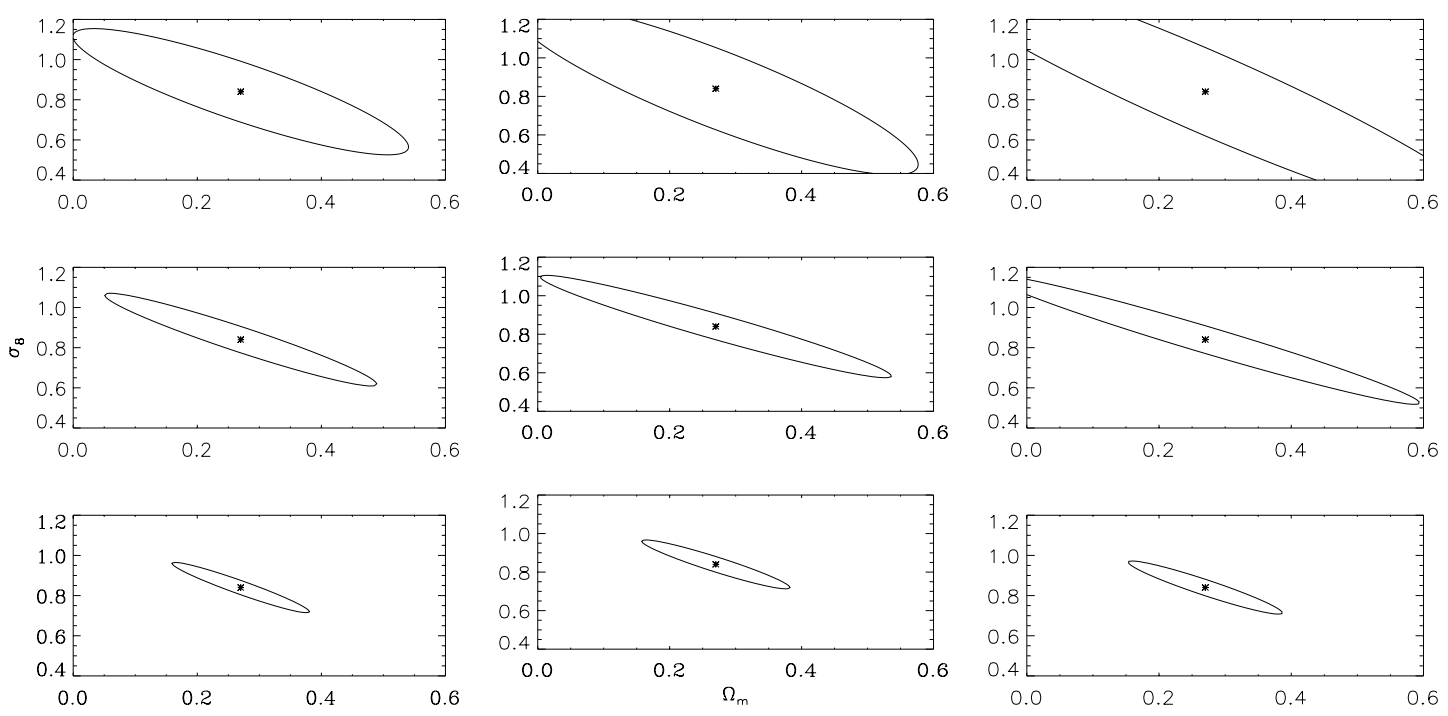

Fig. 3. Constraints on $\sigma_{8}$ and $\Omega_{\mathrm{M}}$ from a joint analysis of the counts, the angular function and the local X-ray temperature function in the presence of gas evolution are shown at one $\sigma$. In the top panel, priors of $30 \%$ on $Y_{*}^{\prime}$ and \pm 1 on $\alpha$ and $\gamma$ have been assumed. In the center panel, the priors on $\alpha$ and $\gamma$ are dropped to \pm 0.1 , with the same prior on $Y_{*}^{\prime}$. In the bottom panel, priors are taken as $15 \%$ on $Y_{*}^{\prime}$ and \pm 0.1 on $\alpha$ and $\gamma$. From left to right, we show the constraints for Planck-like, SPT-like and APEX-like surveys. These constraints are summarized in Table 3.

Table 3. One sigma constraints from a joint analysis of the counts, the angular function and the local X-ray temperature function. For each survey, the prior uncertainties and the final expected constraints on the considered parameters are given. This table summarizes the results of Fig. 3.

\begin{tabular}{ccccc}
\hline \hline Survey & $Y_{*}^{\prime}$ prior unc. $(\%)$ & $\alpha$ and $\gamma$ prior unc. & $\sigma_{\sigma_{8}}(\%)$ & $\sigma_{\Omega_{\mathrm{M}}}(\%)$ \\
\hline Planck & 30 & 1 & 30 & 70 \\
& 30 & 0.1 & 20 & 50 \\
& 15 & 0.1 & 10 & 30 \\
\hline \multirow{2}{*}{ SPT } & 30 & 1 & 40 & 80 \\
& 30 & 0.1 & 20 & 70 \\
& 15 & 0.1 & 10 & 30 \\
\hline APEX & 30 & 1 & 50 & 1 \\
& 30 & 0.1 & 30 & 80 \\
& 15 & 0.1 & 10 & 30 \\
\hline
\end{tabular}

$Y_{*}^{\prime}=0.17 \mathrm{keV}\left(f_{\text {gas }}=0.07 \times h^{-1.5}, h=0.72, T_{*}=1.5 \mathrm{keV}\right)$ and $\alpha=0$ and $\gamma=0$. In the top panel, we assume priors of $30 \%$ on $Y_{*}^{\prime}\left(30 \%\right.$ on $\left.T_{*}\right)$ and \pm 1 on the exponents $\alpha$ and $\gamma$. These latter drop to \pm 0.1 for $\alpha$ and $\gamma$ in the center panel, with the same prior on $Y_{*}^{\prime}$; and in the bottom panel, priors are taken as $15 \%$ on $Y_{*}^{\prime}\left(10 \%\right.$ on $\left.T_{*}\right)$ and \pm 0.1 on $\alpha$ and $\gamma$. The priors and the constraints are summarized in Table 3. Better knowledge of $Y_{*}^{\prime}$ obviously improves the cosmological constraints, as seen in going to the bottom panel of the figure.

Comparison of the top and middle panels of Fig. 3 shows that the constraints are affected by uncertain gas evolution $(\alpha$ and $\gamma$ ). We note, however, that the Planck catalog is less sensitive to this effect, because it primarily includes massive clusters out to redshifts of only order unity.

\subsection{Constraints on cluster physics}

We now suppose that $\sigma_{8}$ and $\Omega_{\mathrm{M}}$ are given and study the gas physics parameters $Y_{*}^{\prime}, \alpha$ and $\gamma$. Once again, we adopt the concordance model with $\sigma_{8}=0.84, \Omega_{\mathrm{M}}=0.27$
(Spergel et al. 2003) and values of $T_{*}=1.5, f_{\mathrm{gas}}=0.07 \mathrm{~h}^{-1.5}$, $\alpha=0$ and $\gamma=0$ for our fiducial model.

Once $\sigma_{8}$ and $\Omega_{\mathrm{M}}$ are given, constraints on $Y_{*}^{\prime}$ can be obtained with just SZ measurements, independently of any other external data, using the counts and the angular correlation function. Adding the constraints on $T_{*}$ from the local X-ray temperature function will place a constraint on $f_{\text {gas }}$ and vice versa when external constraints on $f_{\text {gas }}$ are available, having the SZ derived constraints on $Y_{*}^{\prime}$ permits us to constraint $T_{*}$ independently of X-ray data.

The one-dimensional likelihood function for $Y_{*}^{\prime}$ is given in Fig. 4 for different priors on cosmological parameters and $\alpha$ and $\gamma$. Since $Y_{*}^{\prime}$ is a linear combination of $T_{*}$ and $f_{\text {gas }}$, constraints on $Y_{*}^{\prime}$ translate into constraints on one of these two parameters once the other one is known. For example, in the best case of a limit of $\approx 15 \%$ on $Y_{*}^{\prime}$ and a $10 \%$ prior on $T_{*}$ (resp. $f_{\text {gas }}$ ), $f_{\text {gas }}\left(\right.$ resp. $T_{*}$ ) will be constrained to $20 \%$; if the prior on $T_{*}$ (resp. $f_{\text {gas }}$ ) is known at $5 \%$, the other parameter will be constrained to $15 \%$. The errors on $Y_{*}^{\prime}$ corresponding to this figure are summarized in Table 4. 

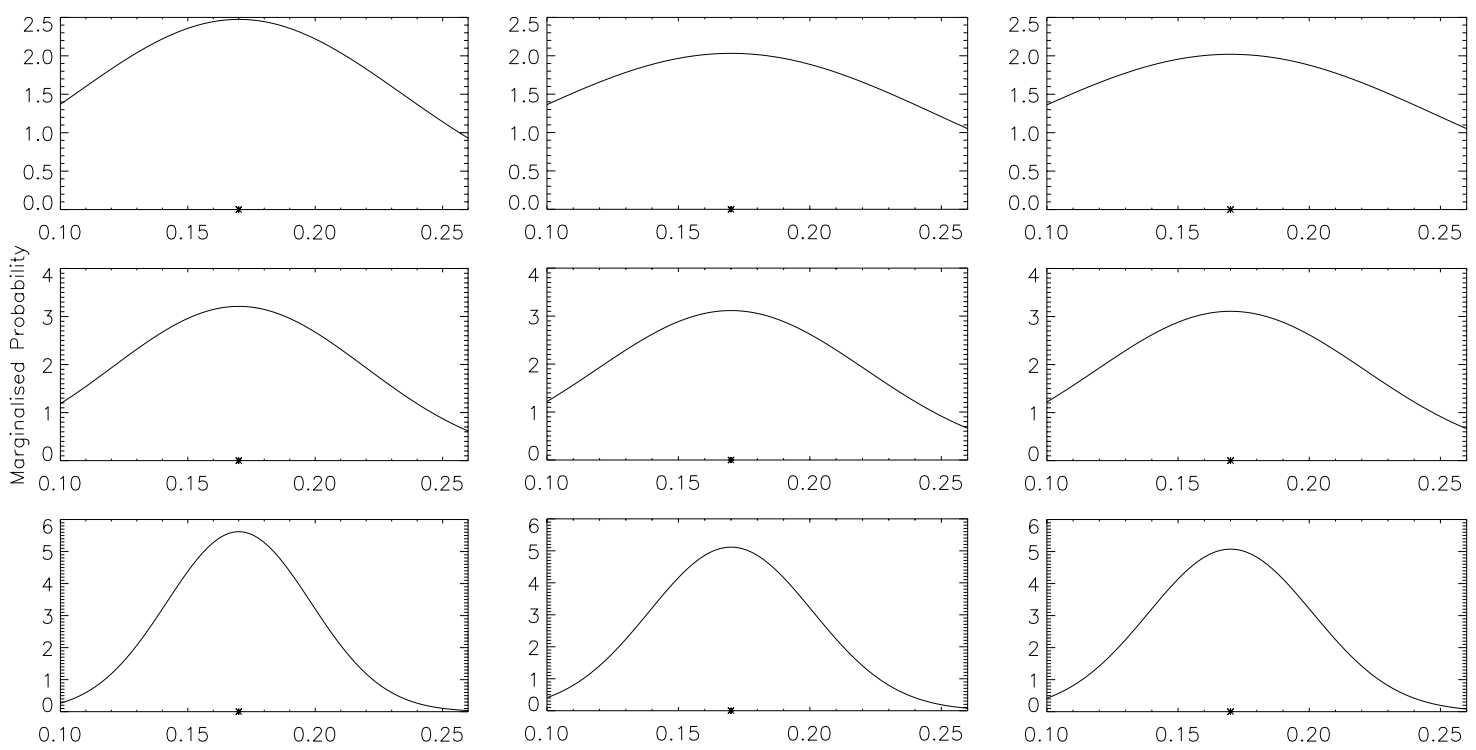

Fig. 4. Constraints on $Y_{*}^{\prime}$ from a joint analysis of the counts and the angular function when the cosmological parameters are known. In the top panel, priors are taken as $10 \%$ on $\sigma_{8}$ and $\Omega_{\mathrm{M}}$, and \pm 1 for $\alpha$ and $\gamma$. In the center panel, the prior on $\alpha$ and $\gamma$ are dropped to \pm 0.1 . In the bottom panel, priors are 5\% on $\sigma_{8}$ and $\Omega_{\mathrm{M}}$, and \pm 0.1 for $\alpha$ and $\gamma$. From left to right, we show the constraints for Planck-like, SPT-like and APEX-like surveys. These constraints are summarized in Table 4.

Table 4. One sigma constraints on $Y_{*}^{\prime}$ from a joint analysis of the counts and the angular function when the cosmological parameters are known. This table corresponds to Fig. 4.

\begin{tabular}{cccc}
\hline \hline Survey & $\alpha$ and $\gamma$ prior unc. & $\sigma_{8}$ and $\Omega_{\mathrm{M}}$ prior unc. $(\%)$ & $\sigma_{Y_{*}^{\prime}}$ \\
\hline Planck & 1 & 10 & 0.5 \\
& 0.1 & 10 & 0.3 \\
& 0.1 & 5 & 0.16 \\
\hline SPT & 1 & 10 & 0.6 \\
& 0.1 & 10 & 0.3 \\
& 0.1 & 5 & 0.18 \\
\hline APEX & 1 & 10 & 0.6 \\
& 0.1 & 10 & 0.3 \\
& 0.1 & 5 & 0.18 \\
\hline
\end{tabular}

This kind of analysis is, on the other hand, unable to constraints the evolution parameters $\alpha$ and $\gamma$; the $\alpha-\gamma$ plane is highly degenerate.

\section{Discussion and conclusion}

Using a Fisher analysis, we have quantified the constraints achievable with a SZ photometric catalog before any subsequent follow-up to obtain redshifts. Our analysis has been restricted to flat cosmologies centered on the concordance model with $\Omega_{\mathrm{M}}=0.27, \sigma_{8}=0.84$ and $h=0.72$. The local abundance of X-ray clusters, as measured by the present-day X-ray temperature distribution function, adds additional information that can be usefully combined with the SZ counts and angular function. With prior information on $T_{*}$, all three parameters $\left(\sigma_{8}, \Omega_{\mathrm{M}}, Y_{*}^{\prime}\right)$ may be constrained, which also yields a constraint on the cluster gas mass fraction $f_{\text {gas }}$. This determination of $f_{\text {gas }}$ would be truly representative of the cluster population, as it is an average over a potentially very large number of objects. Constraints on the order of $10 \%$ to $30 \%$ (around the concordance values) are obtained on the cosmological parameters $\left(\sigma_{8}, \Omega_{\mathrm{M}}\right)$ with both an all-sky survey to $Y \sim 10^{-4} \operatorname{arcmin}^{2}$ and a deep ground-based survey to $Y \sim$ few $\times 10^{-5} \operatorname{arcmin}^{2}$ (see Figs. 2 and 3). To achieve these results, one must have external information equivalent to a $10 \%$ prior on the value of the normalization of the $T-M$ relation $\left(T_{*}\right)$.

These general results are not hugely affected by non-standard (i.e., non self-similar) gas evolution, in particular in the case of Planck. The corollary is that we are unable to turn the argument around in the sense that even if the cosmological parameters are taken as fixed, very little restriction is placed on gas evolution. On the other hand, if the cosmological parameters are known, we are able, by constraining the normalization of the $Y(M, z)$ relation, to constrain the present day $(z=0)$ gas mass fraction $f_{\text {gas }}$ to about $20 \%$ (with a prior of $10 \%$ on the normalisation of the mass/temperature relation $T_{*}$ ); or, vice versa, the normalization of the mass/temperature relation $T_{*}$ to about $20 \%$ (with a prior of $10 \%$ on $f_{\text {gas }}$ ). Once again, this represents a measurement over a very large number of clusters.

In conclusion, an angular SZ catalog in which both the counts and angular correlation function are measured can provide useful cosmological constraints, permitting an immediate return on a SZ survey before subsequent follow-up observations.

Acknowledgements. S. Mei thanks Matthias Bartelmann, Frank Bertoldi and Saleem Zaroubi for useful discussions, and acknowledges support from the European Space Agency External Fellowship program. Some of this work was performed at the Lawrence Berkeley National Laboratory and at the University of California, Berkeley, thanks to funding from the France Berkeley Fund (grant "Precision Cosmology from CMB analysis"). 


\section{References}

Aghanim, N., de Luca, A., Bouchet, F. R., et al. 1997, A\&A, 325, 9 Arnaud, M., Aghanim, N., \& Neumann, D. M. 2002, A\&A, 389, 1

Barbosa, D., Bartlett, J. G., Blanchard, A., et al. 1996, A\&A, 314, 13 Bartelmann, M. 2001, A\&A, 370, 754

Bartelmann, M., \& White, S. D. M. 2002, A\&A, 388, 732

Bartlett, J. G. 2000 [arXiv: astro-ph/0001267]

Benson, A. J., Reichardt, C., \& Kamionkowski, M. 2002, MNRAS, 331,71

Birkinshaw, M. 1999, Phys. Rep., 310, 97

Bryan, G. L., \& Norman, M. L. 1998, ApJ, 495, 80

Carlstrom, J. E., Holder, G. P., \& Reese, E. D. 2002, ARA\&A, 40, 643

Carroll, S. M., Press, W. H., \& Turner, E. L. 1992, ARA\&A, 30, 499

Colafrancesco, S., Mazzotta, P., \& Vittorio, N. 1997, ApJ, 488, 566

da Silva, A. C., Kay, S. T., Liddle, A. R., et al. 2004, MNRAS, 348, 1401

Diaferio, A., Nusser, A., Yoshida, N., et al. 2003, MNRAS, 338, 433

Diego, J. M., Martínez-González, E., Sanz, J. L., et al. 2002, MNRAS, 331,556

Eke, V. R., Cole, S., \& Frenk, C. S. 1996, MNRAS, 282, 263

Eisenstein, D. J., Hu, W., \& Tegmark, M. 1999, ApJ, 518, 2

Ettori, S., Tozzi, P., Borgani, S., et al. 2004, A\&A, 417, 13

Fan, Z., \& Chiueh, T. 2001, ApJ, 550, 547

Finoguenov, A., Reiprich, T. H., \& Böhringer, H. 2001, A\&A, 368, 749

Grego, L., Carlstrom, J. E., Reese, E. D., et al. 2002, ApJ, 552, 2

Haiman, Z., Mohr, J. J., \& Holder, G. 2001, ApJ, 553, 545

Holder, G., Haiman, Z., \& Mohr, J. J. 2001, 560, L111

Horner, D. J., Mushotsky, R. F., \& Scharf, C. A. 1999, ApJ, 520, 78

Hu, W. 2003, Ph. Rv. D, 67, 1304

Huterer, D., \& White, M. 2002, ApJ, 578, L95
Kaiser, N. 1986, MNRAS, 222, 323

Kneissl, R., Jones, M. E., Saunders, R., et al. 2001, MNRAS, 328, 783

Landy, S. D., \& Szalay, A. S. 1993, ApJ, 412, 64

Levine, E. S., Schulz, A. E., \& White, M. 2002, ApJ, 577, 569

Majumdar, S., \& Mohr, J. J. 2003a, ApJ, 585, 603

Majumdar, S., \& Mohr, J. J. 2003b [arXiv:astro-ph/0305341]

Mei, S., \& Bartlett, J. G. 2003, A\&A, 410, 767 (MB03)

Mohr, J. J., Mathiesen, B., \& Evrard, A. E. 1999, ApJ, 517, 627

Moscardini, L., Bartelmann, M., Matarrese, S., et al. 2002, MNRAS, 340,102

Muanwong, O., Thomas, P. A., Kay, S. T., et al. 2002, MNRAS, 336, 527

Nevalainen, J., Markevitch, M., \& Forman, W. 2000, ApJ, 532, 694

Oukbir, J., \& Blanchard, A. 1997, A\&A, 317, 1

Peacock, J. A., \& Dodds, S. J. 1996, MNRAS, 280, L19

Peebles, P. J. E. 1980, The Large-Scale Structure of the Universe, Princeton Series in Physics (New Jersey: Princeton, Princeton University Press)

Pierpaoli, E., Borgani, S., Scott, D., et al. 2003, MNRAS, 342,163

Press, W. H., Teukolsky, S. A., Vetterling, W. T., Flannery, B. P., et al. 1992, Numerical Recipes (New York: Cambridge University Press)

Sheth, R. K., \& Tormen, G. 1999, MNRAS, 308, 199

Sheth, R. K., Mo, H. J., \& Tormen, G. 2001, MNRAS, 323, 1

Spergel, D. N., Verde, L., Peiris, H. V., et al. 2003, ApJS, 148, 175S

Sunyaev, R. A., \& Zel'dovich, Ya. B. 1970, Comments Astrophys. Space Phys., 2, 66

Sunyaev, R. A., \& Zel'dovich, Ya. B. 1972, Comments Astrophys. Space Phys., 4, 173

Weller, J., Battye, R. A., \& Kneissl, R. 2002, Phys. Rev. Lett., 88, 1301

Xu, H., Jin, G., \& Wu, X.-P. 2001, ApJ, 553, 78 